\title{
Incidence, clinical features, and risk factors of fluoroquinolone-induced acute liver injury: a case-control study
}

This article was published in the following Dove Medical Press journal:

Therapeutics and Clinical Risk Management

\section{Hong-Yi Yang \\ Dai-Hong Guo \\ Wang-Ping Jia \\ Man Zhu \\ Yuan-Jie Xu \\ Xiao-Yu Wang}

Department of Pharmaceutical

Care, General Hospital of People's

Liberation Army, Beijing I00853, China

Correspondence: Dai-Hong Guo Department of Pharmaceutical Care, General Hospital of People's Liberation Army, 28 Fu Xing Road, Beijing 100853, China

Tel +86 I39 I0I5 3966

Email guodh30I@I26.com
Background: Fluoroquinolone-related hepatotoxicity is rare but serious and is attracting increasing attention. We explored the incidence, clinical features and risk factors of acute liver injury associated with fluoroquinolone use.

Materials and methods: Based on the Adverse Drug Events Active Surveillance and Assessment System that we developed, we carried out a case-control study by enrolling patients who were hospitalized and received fluoroquinolones to treat or prevent infections at the Chinese People's Liberation Army General Hospital from Jan 2016 to Dec 2017. The incidence of fluoroquinolone-induced acute liver injury was estimated, and logistic regression was used to reveal the risk factors of this adverse reaction.

Results: We found that 17,822 patients received fluoroquinolones, and 13,678 of them met the inclusion criteria. A total of 91 patients developed acute liver injury after receiving the medication, and 369 controls were matched to these patients. The overall incidence of fluoroquinoloneinduced acute liver injury in the Chinese population is approximately 6-7 cases per 1,000 individuals annually. Multivariate logistic regression analysis showed that older age slightly decreased the risk of hepatotoxicity (OR, 0.98; 95\% CI, 0.96-0.99). The male sex (OR, 2.19; 95\% CI, 1.07-4.48), alcohol abuse (OR, 2.91; 95\% CI, 1.39-6.11) and hepatitis B carrier status $(\mathrm{OR}, 2.38 ; 95 \% \mathrm{CI}, 1.04-5.48)$ increased the risk of liver injury. Concurrent use of cephalosporins or carbapenems was also associated with an increased risk.

Conclusion: Increased risk of fluoroquinolone-related hepatotoxicity may be associated with youth, the male sex, alcohol abuse, hepatitis B carrier status and the concurrent use of cephalosporins or carbapenems.

Keywords: fluoroquinolones, drug-induced liver injury, incidence, risk factors, pharmacovigilance

\section{Introduction}

Fluoroquinolones have been widely used in clinical practice for over 20 years because of their broad-spectrum antibacterial activity and excellent bioavailability. ${ }^{1}$ Despite their effectiveness, fluoroquinolones are also associated with several rare but serious adverse reactions, including arthropathy, QT interval prolongation, neurological events, phototoxicity and retinal detachment. In addition, idiosyncratic reactions related to fluoroquinolones, especially hepatotoxicity, have become an increasing concern. ${ }^{1-4}$

Moxifloxacin is a relatively new antibacterial agent with improved activity against gram-positive bacteria and anaerobes and has equal or higher bioavailability and a longer elimination half-life than the previous fluoroquinolones. ${ }^{5,6}$ However, moxifloxacin is not exempt from safety concerns, especially the potential risk of hepatotoxicity. ${ }^{7,8}$ The Medicines and Healthcare products Regulatory Agency issued a 
restriction on the use of moxifloxacin in 2011 requiring that oral moxifloxacin should be used only when in cases that are unsuitable for treatment with other antibacterial agents that are commonly recommended for the initial treatment or when other treatments fail. The same year, the China Food and Drug Administration also warned of liver injury related to moxifloxacin use. One of the most frequently prescribed fluoroquinolones, levofloxacin, also carries a risk of inducing liver injury, and its hepatotoxicity has already been reported many times. ${ }^{9,10}$ Thus, it is important for health care providers to be aware of the association of fluoroquinolones with hepatotoxicity.

Unfortunately, there are few published studies about fluoroquinolone-induced acute liver injury, especially in the Chinese population. Therefore, based on the Adverse Drug Events Active Surveillance and Assessment System (ADE-ASAS) developed by the People's Liberation Army (PLA) Adverse Drug Reaction Monitoring Center, we conducted a case-control study to explore the incidence, clinical features, and risk factors of acute liver injury associated with fluoroquinolone use.

\section{Materials and methods Study design and data source}

We carried out a case-control study to research the characteristics of fluoroquinolone-induced acute liver injury in a cohort of inpatients over 18 years old. The patients were admitted to the Chinese PLA General Hospital and received fluoroquinolones to treat or prevent infections from Jan 2016 to Dec 2017. The patients' blood was drawn and tested over approximately 2-3 days.

Patient data, including demographics, diagnoses, prescriptions, laboratory test results and outcomes, were recorded in the Hospital Information System (HIS). We developed ADE-ASAS based on trigger technology, which can be connected to the HIS and extract information of patients from it. Based on the inclusion and exclusion triggers of the liver injury module, which we set in advance, the system could preliminarily determine whether patients developed liver injury and send warning signals once the monitoring indicators reached the criteria for drug-induced liver injury (DILI). Finally, two clinical pharmacists performed backto-back evaluations to identify the cases. If patients had multiple positive signals during hospitalization, only the first signal was assessed. The accuracy of this system was evaluated in multiple studies with a positive predictive value up to $69.4 \% .{ }^{11,12}$ This study complied with the Declaration of Helsinki and was approved by the Medical Ethics Committee of the Chinese PLA General Hospital. Patient consent to review their medical records was not required because this was an observational study that did not infringe upon the interests or rights of the patients. All patient data were kept strictly confidential.

\section{Identification of cases and controls}

Information on patients who were hospitalized and received fluoroquinolones between Jan 2016 and Dec 2017 was monitored and extracted from the HIS using ADE-ASAS. Patients with abnormal or no laboratory baseline data and patients who were diagnosed with liver injury or liver failure before treatment (exclusion triggers) were automatically excluded. According to clinical chemistry criteria issued by the International Serious Adverse Event Consortium, the inclusion triggers were set as follows: 1 ) ALT $\geq 5$ times the upper limit of normal (ULN), 2) ALP $\geq 2$ times the ULN (particularly with accompanying elevations in the concentrations of $5^{\prime}$-nucleotidase or $\gamma$-glutamyl transpeptidase in the absence of known bone pathology driving the rise in the ALP level), or 3) ALT $\geq 3$ times the ULN and total serum bilirubin (TBil) $\geq 2$ times the ULN. ${ }^{13}$

Two clinical pharmacists independently assessed the included subjects who had warning signals and excluded patients who had histories of liver or bone marrow transplants or were suspected to have a paracetamol overdose. Some chronic liver diseases (such as alcoholic liver diseases) and serologies for acute hepatitis A, hepatitis B, cytomegalovirus and Epstein-Barr virus were also considered. ${ }^{3,14}$ The Roussel Uclaf Causality Assessment Method scale was used to evaluate the causal relationship between fluoroquinolones and acute liver injury. Cases with total score of 3 (possible) or above were categorized as the case group. Clinical severity of DILI was classified as follows: mild, elevated ALT or ALP concentrations reaching the criteria for DILI but TBil concentration $<2$ times the ULN; moderate, elevated ALT or ALP concentrations reaching the criteria for DILI and TBil concentration $\geq 2$ times the ULN, or symptomatic hepatitis; severe, elevated ALT or ALP concentrations reaching the criteria for DILI, TBil concentration $\geq 2$ times the ULN, and one of the following: 1) international normalized ratio $\geq 1.5,2$ ) ascites and/or encephalopathy, disease duration $<26$ weeks, and absence of underlying cirrhosis, or 3 ) other organ failure considered to be due to DILI. ${ }^{13}$ The pattern of liver injury was classified as hepatocellular, cholestatic or mixed by the ratio of abnormalities in liver function (R), which compares ALT and ALP in multiples 
of their ULN. $R>5$ was considered hepatocellular; $<2$, cholestatic; and 2-5, mixed. ${ }^{3,14,15}$

We randomly selected controls from the patients who received fluoroquinolones but did not have enzymes elevation or clinical symptoms. Up to four controls were matched to each case according to the time of admission and primary diagnosis because they had similar conditions and therapeutic schedules to those of the cases.

\section{Statistical analysis}

The demographic profiles and clinical information for the case and control groups were collected. We used chi-square or Fisher's exact tests to analyze categorical data and used Student's $t$-test or the Mann-Whitney $U$ test for continuous data.
The risk factors of fluoroquinolone-induced acute liver injury were determined via univariate and multivariate binary logistic regression analysis. The unadjusted OR and 95\% CI were calculated for each variable in the univariate logistic regression, and multiple logistic regression $(P<0.05$ was used for entry and $>0.10$ for removal) was used to assess independent risk factors of fluoroquinolone-induced acute liver injury. The adjusted OR and 95\% CI were calculated for each included variable. Potential confounding factors included age, sex, BMI, alcohol abuse, smoking, allergy history, serum albumin, hemoglobin, the status of hepatitis B virus (HBV), comorbidities (diabetes, hypertension, cardiovascular disease) and concurrent use of other drugs. ${ }^{16,17}$ The complete study process is presented in Figure 1.

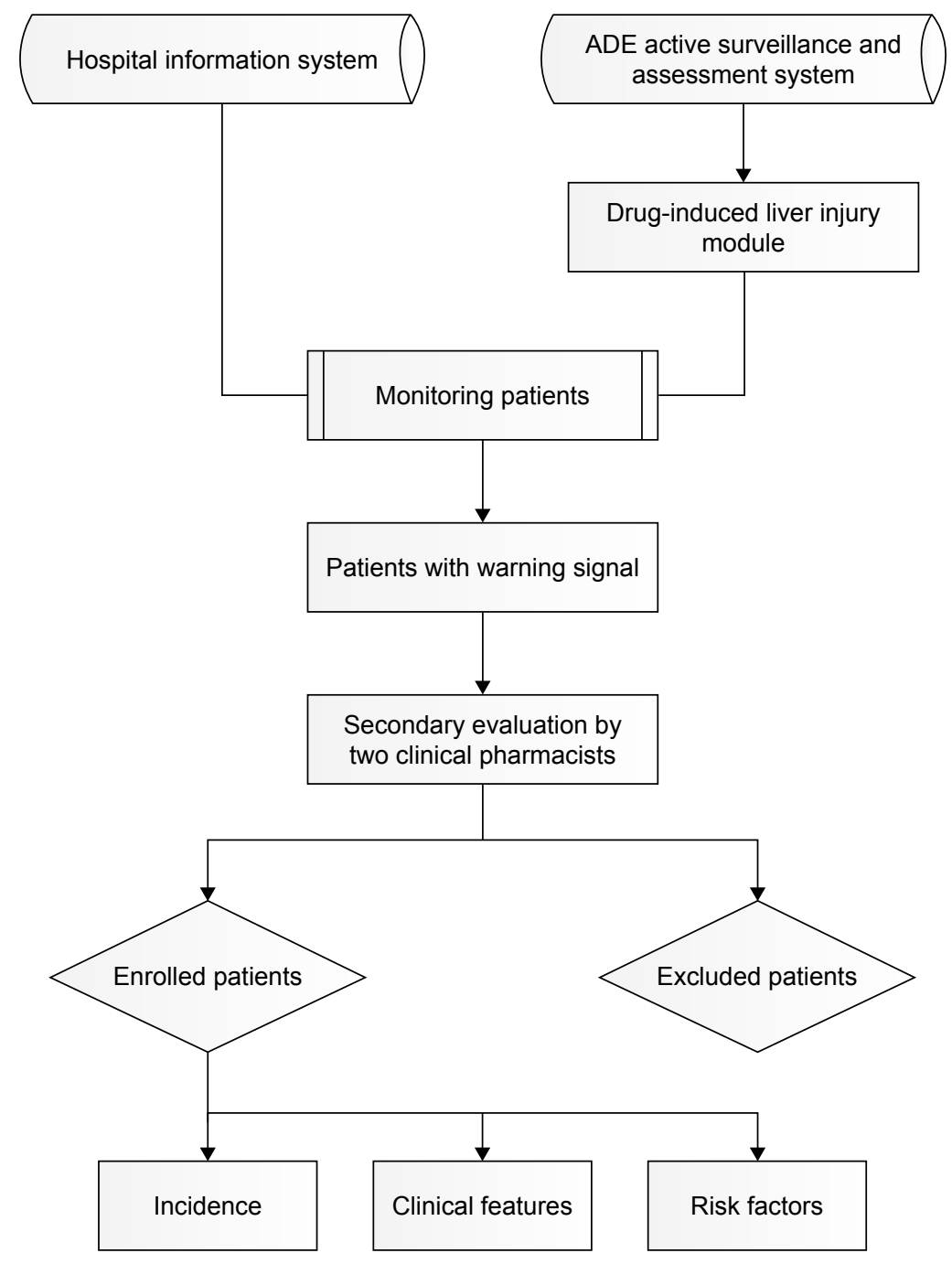

Figure I The technical flow chart of this study.

Notes: The information on patients using fluoroquinolones was extracted and preliminarily analyzed by the ADE active surveillance and assessment system from the hospital information system. After the secondary evaluation by two clinical pharmacists, the incidence, clinical features and risk factors of fluoroquinolone-induced acute liver injury were researched.

Abbreviation: $A D E$, adverse drug events. 
All statistical analyses were performed using SPSS software, version 19.0 (SPSS Inc., Chicago, IL, USA). A two-sided $P$-value less than 0.05 was set as the threshold for statistical significance.

\section{Results}

We monitored and found 17,822 patients who received fluoroquinolones to prevent or treat infections. Of these patients, 4,144 (23.25\%) were automatically excluded by ADE-ASAS, and warning signals were sent for 657 (4.80\%) of the remaining patients. Finally, after back-to-back revaluation by two clinical pharmacists, we identified 91 patients who developed liver injury, and 369 controls were matched to these patients. The positive predictive value of ADE-ASAS in this study was only $13.85 \%$ because the exclusion triggers of the system were set loosely to increase the sensitivity so that the results could better reflect the real-world characteristics of fluoroquinolone-induced acute liver injury. On the other hand, the hepatotoxicity diagnoses must include other necessary examinations and the estimate of causality by pharmacists in addition to biochemical tests. Nevertheless, the system saved quite a lot of manpower and material resources.

In the case group, hepatitis occurred during the treatment period for 42 patients $(46.15 \%)$ and after cessation of therapy for 49 patients (53.85\%). Among these patients, hepatitis was identified as mild in 69 patients $(75.82 \%)$, moderate in seven patients $(7.70 \%)$ and severe in 15 patients $(16.48 \%)$ ). Regarding the pattern of hepatitis, 41 patients $(45.06 \%)$ were diagnosed with hepatocellular injury, 35 patients (38.46\%) were diagnosed with cholestatic injury, and 15 patients (16.48\%) were diagnosed with mixed injury. The median time from fluoroquinolones use to developing acute liver injury was 7 days (IQR 4-12). Only four patients (4.40\%) developed jaundice during and after therapy. Fever was observed in 21 patients (23.08\%). Nine patients $(9.89 \%)$ had eosinophilia, and two patients (2.20\%) experienced skin rash. No patients developed encephalopathy. After intervention therapy (such as for drug withdrawal and prescriptions of hepatoprotectant), 72 patients $(79.12 \%)$ recovered from liver injury, 18 patients $(19.78 \%)$ had poor outcomes (liver enzymes and bilirubin levels remained unchanged or continued to rise), and one patient $(1.10 \%)$ died of liver failure.

The demographic and clinical characteristics of the cases and controls are presented in Table 1. In the case group, 21 patients $(23.1 \%)$ were younger than or equal to 44 years old, 29 patients (31.9\%) were between 45 and 59 years old, and 41 patients (45.0\%) were 60 years or older. In the control group, 68 patients (18.4\%) were aged 44 or younger,
Table I Characteristics of cases and controls

\begin{tabular}{|c|c|c|c|}
\hline \multirow[t]{2}{*}{ Variable } & \multicolumn{2}{|c|}{$\begin{array}{l}\text { No. (\%) of patients or } \\
\text { mean } \pm \text { SD }\end{array}$} & \multirow[t]{2}{*}{$P$-value } \\
\hline & $\begin{array}{l}\text { Cases } \\
(n=91)\end{array}$ & $\begin{array}{l}\text { Controls } \\
(\mathrm{n}=369)\end{array}$ & \\
\hline Age (year) & $56.32 \pm 18.47$ & $58.97 \pm 16.10$ & 0.17 \\
\hline Sex (male) & $69(75.8)$ & $195(52.8)$ & $<0.01$ \\
\hline BMI $\left(k g / m^{2}\right)$ & $22.54 \pm 3.83$ & $23.95 \pm 4.02$ & $<0.01$ \\
\hline Albumin (g/L) & $34.3 I \pm 5.10$ & $35.47 \pm 4.73$ & 0.04 \\
\hline Hemoglobin (g/L) & $107.82 \pm 25.95$ & || $3.5 \mid \pm 20.70$ & 0.05 \\
\hline Alcohol abuse & $31(34.1)$ & $40(10.8)$ & $<0.01$ \\
\hline Smoking & & & $<0.01$ \\
\hline Never & $45(49.4)$ & $253(68.6)$ & \\
\hline Current & $33(36.3)$ & $53(14.4)$ & \\
\hline Past & $13(14.3)$ & $63(17.0)$ & \\
\hline Allergy history & $25(27.5)$ & $81(22.0)$ & 0.27 \\
\hline \multicolumn{4}{|l|}{ Comorbidities } \\
\hline Hypertension & $31(34.1)$ & $128(34.7)$ & 1.00 \\
\hline Diabetes mellitus & $16(17.6)$ & $64(17.3)$ & 1.00 \\
\hline $\begin{array}{l}\text { Cardiovascular } \\
\text { disease }\end{array}$ & $14(15.4)$ & $64(17.3)$ & 0.76 \\
\hline Hepatitis B carrier & $14(15.4)$ & $23(6.2)$ & $<0.01$ \\
\hline Combination drug & & & $<0.01$ \\
\hline $\begin{array}{l}\text { Proton pump } \\
\text { inhibitors }\end{array}$ & $75(82.4)$ & $238(64.5)$ & \\
\hline Cephalosporins & $42(46.2)$ & $107(29.0)$ & \\
\hline Carbapenems & $33(36.3)$ & $51(13.8)$ & \\
\hline Antifungal agents & $23(25.3)$ & $38(10.3)$ & \\
\hline Aminoglycosides & $7(7.7)$ & $23(6.2)$ & \\
\hline
\end{tabular}

Note: ${ }^{2} \chi^{2}$ or $t$-test, as appropriate.

117 patients (31.7\%) were aged 45-59, and 184 (49.9\%) patients were older than 60 years. The percentage of women was significantly higher in the control group than in the case group (47.2\% vs $24.2 \%$ ). Control patients had higher BMIs and higher levels of albumin than case patients (23.95 vs 22.54 and 35.47 vs 34.31 , respectively). Cases were more likely than controls to smoke ( $36.3 \%$ vs $14.4 \%$ ) and abuse alcohol (34.1\% vs $10.8 \%)$. Relative to the control group, the case group had a greater proportion of patients carrying hepatitis B virus (15.4\% vs $6.2 \%)$ and receiving combination drug therapy.

The findings from univariate and multivariate analyses of the risk factors are presented in Table 2. There were many independent risk factors of fluoroquinolone-induced liver injury, including age, sex, alcohol abuse, hepatitis B carrier status and concurrent use of other drugs. Relative to younger patients, older patients had slightly decreased the risk for liver injury after adjusting for all confounders (OR, 0.98; 95\% CI, 0.96-0.99). Men had a greater probability of developing hepatotoxicity (OR, 2.19; 95\% CI, 1.07-4.48). Furthermore, we found a 2.91-fold increased risk of developing hepatotoxicity in the patients abusing 
Table 2 Univariate and multivariate analyses of risk factors of fluoroquinolone-induced acute liver injury

\begin{tabular}{|c|c|c|}
\hline Variable & $\begin{array}{l}\text { Unadjusted } \\
\text { OR }(95 \% \mathrm{Cl})\end{array}$ & $\begin{array}{l}\text { Adjusted } \\
\text { OR }(95 \% \mathrm{Cl}) *\end{array}$ \\
\hline Age (year) & $0.99(0.98-1.00)$ & $0.98(0.96-0.99)$ \\
\hline Sex (male) & $2.80(1.66-4.72)$ & $2.19(1.07-4.48)$ \\
\hline \multicolumn{3}{|l|}{ BMI $\left(\mathrm{kg} / \mathrm{m}^{2}\right)$} \\
\hline$<18.5$ & $2.30(1.15-4.60)$ & $2.32(1.00-5.39)$ \\
\hline$\geq 18.5$ & I & 1 \\
\hline \multicolumn{3}{|l|}{ Albumin (g/L) } \\
\hline$\leq 25$ & $2.94(0.68-12.7)$ & $4.02(0.72-22.31)$ \\
\hline $26-34$ & $1.57(0.97-2.52)$ & $1.22(0.68-2.17)$ \\
\hline$\geq 35$ & I & 1 \\
\hline \multicolumn{3}{|l|}{ Hemoglobin (g/L) } \\
\hline$<110$ & $1.59(1.01-2.53)$ & $1.38(0.77-2.48)$ \\
\hline$\geq 110$ & I & I \\
\hline Alcohol abuse & $4.25(2.47-7.32)$ & $2.91(1.39-6.11)$ \\
\hline \multicolumn{3}{|l|}{ Smoking } \\
\hline Never & 1 & 1 \\
\hline Current & $3.50(2.04-6.00)$ & $1.91(0.79-4.61)$ \\
\hline Past & $1.16(0.59-2.28)$ & $2.33(0.98-5.55)$ \\
\hline Allergy history & $1.35(0.80-2.27)$ & $1.36(0.74-2.49)$ \\
\hline \multicolumn{3}{|l|}{ Comorbidities } \\
\hline Hypertension & $0.97(0.60-1.58)$ & $1.18(0.63-2.23)$ \\
\hline Diabetes mellitus & $1.02(0.56-1.86)$ & $0.91(0.42-1.97)$ \\
\hline Cardiovascular disease & $0.87(0.46-1.63)$ & $1.46(0.64-3.32)$ \\
\hline Hepatitis B carrier & $2.74(1.35-5.56)$ & $2.38(1.04-5.48)$ \\
\hline \multicolumn{3}{|l|}{ Combination drug } \\
\hline Proton pump inhibitors & $6.41(1.95-21.02)$ & $3.33(0.92-12.06)$ \\
\hline Cephalosporins & 7.98 (2.37-26.84) & $1.84(1.06-3.21)$ \\
\hline Carbapenems & $13.16(3.8 \mid-45.42)$ & $2.53(1.35-4.73)$ \\
\hline Antifungal agents & $|2.3|(3.46-43.80)$ & $2.02(1.00-4.06)$ \\
\hline Aminoglycosides & $6.19(1.47-25.99)$ & $0.90(0.32-2.52)$ \\
\hline
\end{tabular}

Note: *Adjusted for all variables in the table.

alcohol compared with other patients (OR, 2.91; 95\% CI, 1.39-6.11). The risk was significantly increased in patients who carried hepatitis B (OR, 2.38; 95\% CI, 1.04-5.48). For all the concurrently used drugs we analyzed, cephalosporins and carbapenems use were two independent risk factors that increased the hepatotoxicity risk 1.84 -fold and 2.53-fold, respectively (OR, 1.84; 95\% CI, 1.06-3.21 and OR, 2.53; 95\% CI, 1.35-4.73). However, other factors, including BMI, albumin, hemoglobin, smoking, allergy history, hypertension, diabetes mellitus, cardiovascular disease and other concurrently used drugs, were not associated with the development of fluoroquinolone-induced acute liver injury.

\section{Discussion}

The epidemiology of DILI in China has rarely been studied before, much less the hepatotoxicity of fluoroquinolones. The crude incidence of hospital admission for DILI has been reported to range from 6 to 9 cases per 100,000 exposures, and this rate is even lower in some spontaneous reports and case-control analyses, which had values ranging from 1 to 6 per million prescriptions. ${ }^{18,19}$ In this study, we found that the overall incidence of fluoroquinolone-induced acute liver injury in the Chinese population was approximately 6-7 cases per 1,000 individuals annually. This result is different from those of previous studies, and one of the possible reasons is that other studies enrolled outpatients who were admitted to the hospital for acute liver injury. This enrollment process might ignore or miss many undiagnosed or symptomless patients. However, the system that we used can monitor mostly inpatients and is less likely to miss probable cases. On the other hand, inpatients have more confounding factors, which may increase their hepatotoxicity risk.

More than half the patients in this study developed liver injury within 7 days after receiving fluoroquinolones, which is consistent with some fluoroquinolone-induced liver injury reports. ${ }^{20-22}$ Although the mechanism of DILI is not well understood, many reports suggest that this adverse reaction could be a hypersensitivity reaction mediated by the immune system, which could be applicable to fluoroquinolones because the reaction occurred quickly and had some immunoallergic features. ${ }^{23,24}$

In our study, advanced age was associated with a slightly lower risk of liver injury, although it is usually considered a cause of increased risk in many other diseases. This result is consistent with the findings of several previous studies. ${ }^{16,25}$ Young age increases susceptibility to DILI, which may be related to drug specificity. For example, valproate and propylthiouracil are more likely to cause severe and fatal hepatotoxicity in children, but isoniazid, amoxicillin-clavulanate and nitrofurantoin increase the risk of liver injury in the elderly population. ${ }^{26}$ In addition, we found that being male nearly doubles the risk for hepatotoxicity. Many studies have shown a preponderance of DILI in women, ${ }^{27-29}$ while some other studies have also shown no significant difference between men and women; ${ }^{30,31}$ thus, whether men or women are at a higher risk for "all-cause DILI" remains inconclusive.

In this study, malnutrition was defined as BMI $<18.5 \mathrm{~kg} / \mathrm{m}^{2}$, anemia was defined as hemoglobin $<110 \mathrm{~g} / \mathrm{L}$, severe and mild albumin loss were defined as albumin $\leq 25$ and 26-34 g/L, respectively. Although poor nutrition status and anemia were found to increase the risk of hepatotoxicity in univariate analysis, no significant correlations between albumin, hemoglobin and hepatotoxicity were detected after multivariate analysis. Malnutrition tends to increase the risk of liver injury; thus, studies with larger sample sizes should be performed to clarify the relationship between these factors. Alcohol dependence was another independent risk factor that 
increased the hepatotoxicity risk by 2.91 -fold, which was consistent with the findings of a previous study, ${ }^{17}$ possibly because alcohol can damage liver cells and may interact with the drugs, which may aggravate the occurrence of liver injury.

In this study, we found a 2.38-fold increased risk of fluoroquinolone-induced acute liver injury in hepatitis B carriers. This finding is consistent with many previous studies that investigated the association between antituberculosis drugs and DILI and suggested that HBV carriers tend to develop liver injury. One study showed that the virus may pose a danger and that the altered cytokine milieu resulting from chronic viral diseases could affect hepatic immunity. This would subsequently contribute to breaking down the immune tolerance in conjunction with drug-induced cellular stress, ${ }^{32}$ so liver function should be closely monitored in hepatitis B patients who simultaneously receive fluoroquinolones to treat infections.

Fluoroquinolones are often prescribed in combination with other drugs and may modulate the hepatotoxic potential of fluoroquinolones by inducing or inhibiting the metabolism through cytochrome P-450 or competing at the level of membrane transporters. ${ }^{33}$ For all the concurrently used drugs we analyzed, cephalosporins and carbapenems use were associated with an increased risk of hepatotoxicity. One recent study has revealed this risk of cephalosporins, and the package inserts for both cephalosporins and carbapenems have described elevations in hepatic aminotransferases. ${ }^{34}$ However, the interaction between cephalosporins, carbapenems and fluoroquinolones is still unclear; therefore, we cannot confirm whether the risk of acute liver injury is increased by the hepatotoxicity of these drugs or their interactions.

This study had some limitations. Due to the wide range of presentations and culprit agents and the lack of objective diagnostic tests, DILI is diagnosed based on exclusion methods, and all other possible factors must be ruled out before making a definitive diagnosis. ${ }^{26}$ However, several clinical characteristics may be missed and can lead to misdiagnoses, such as autoimmune or genetic diseases, and we may not completely exclude the patients with these conditions. Consequently, the incidence of this important adverse reaction may be overestimated and requires further confirmation.

Apart from the above risk factors, several human leukocyte antigen (HLA) serotypes have been identified as genetic risk factors of DILI (flucloxacillin with $B * 57: 01$, and amoxicillin-clavulanate with $A^{*} 02: 01$ ), suggesting an important immunologic component to DILI. ${ }^{33,35}$ However, two published genome-wide association studies found no significant signals for fluoroquinolone-induced liver injury and no evidence that there is an HLA association was obtained..$^{36,37} \mathrm{We}$ thought this form of DILI may not be HLA-related, and these confounders were not included when we used multivariate models to adjust for other nongenetic factors.

Finally, the enrolled patients were all from the Chinese PLA General Hospital, so their characteristics have limitations in the generalizability and representativeness. Further comprehensive and multicenter studies are urgently needed to confirm our findings and define the pathogenesis of this adverse reaction.

\section{Conclusion}

This study indicated that increased risks of fluoroquinoloneinduced acute liver injury may be associated with youth, the male sex, alcohol abuse, hepatitis B carrier status and the concurrent use of cephalosporins or carbapenems, although the results require confirmation in further studies. Physicians should frequently examine the liver function of patients when the above factors are present.

\section{Acknowledgments}

This study was supported by the National Natural Science Foundation of China (Grant No. 81773825). The funding body had no role in the study design, data collection, data analysis, interpretation, or manuscript writing.

\section{Disclosure}

The authors report no conflicts of interest in this work.

\section{References}

1. Sousa J, Alves G, Fortuna A, Falcao A. Third and fourth generation fluoroquinolone antibacterials: a systematic review of safety and toxicity profiles. Curr Drug Saf. 2014;9(2):89-105.

2. Owens RC, Ambrose PG. Antimicrobial safety: focus on fluoroquinolones. Clin Infect Dis. 2005;41(Suppl 2):S144-S157.

3. Orman ES, Conjeevaram HS, Vuppalanchi R, et al. Clinical and histopathologic features of fluoroquinolone-induced liver injury. Clin Gastroenterol Hepatol. 2011;9(6):517-523.e3.

4. Licata A, Randazzo C, Morreale I, Butera G, D’Alessandro N, Craxì A. Fluoroquinolone-induced liver injury: three new cases and a review of the literature. Eur J Clin Pharmacol. 2012;68(5):525-532.

5. Duggirala A, Joseph J, Sharma S, Nutheti R, Garg P, Das T. Activity of newer fluoroquinolones against gram-positive and gram-negative bacteria isolated from ocular infections: an in vitro comparison. Indian J Ophthalmol. 2007;55(1):15-19.

6. Lubasch A, Keller I, Borner K, Koeppe P, Lode H. Comparative pharmacokinetics of ciprofloxacin, gatifloxacin, grepafloxacin, levofloxacin, trovafloxacin, and moxifloxacin after single oral administration in healthy volunteers. Antimicrob Agents Chemother. 2000;44(10):2600-2603.

7. Soto S, López-Rosés L, Avila S, et al. Moxifloxacin-induced acute liver injury. Am J Gastroenterol. 2002;97(7):1853-1854. 
8. Kaye JA, Castellsague J, Bui CL, et al. Risk of acute liver injury associated with the use of moxifloxacin and other oral antimicrobials: a retrospective, population-based cohort study. Pharmacotherapy. 2014; 34(4):336-349.

9. Levine C, Trivedi A, Thung SN, Perumalswami PV. Severe ductopenia and cholestasis from levofloxacin drug-induced liver injury: a case report and review. Semin Liver Dis. 2014;34(2):246-251.

10. Gulen M, Ay MO, Avci A, Acikalin A, Icme F. Levofloxacin-induced hepatotoxicity and death. Am J Ther. 2015;22(3):e93-e96.

11. Chen C, Jia W, Guo D, et al. Development of a computer-assisted adverse drug events alarm and assessment system for hospital inpatients in China. Ther Innov Regul Sci. 2018;18:216847901881019.

12. Guo D, Chang SU, Wang X, et al. Research and practice of Informationalized automatic monitoring on inpatients with Drug-induced liver injury. Eval Anal Drug-Use Hosp China. 2017;17:1019-1021.

13. Aithal GP, Watkins PB, Andrade RJ, et al. Case definition and phenotype standardization in drug-induced liver injury. Clin Pharmacol Ther. 2011; 89(6):806-815.

14. Fontana RJ, Watkins PB, Bonkovsky HL, et al. Drug-induced liver injury network (DILIN) prospective study: rationale, design and conduct. Drug Saf. 2009;32(1):55-68.

15. Kim SH, Naisbitt DJ. Update on advances in research on idiosyncratic drug-induced liver injury. Allergy Asthma Immunol Res. 2016;8(1):3-11.

16. Alshammari TM, Larrat EP, Morrill HJ, Caffrey AR, Quilliam BJ, Laplante KL. Risk of hepatotoxicity associated with fluoroquinolones: a National Case-Control Safety Study. Am J Health Syst Pharm. 2014; 71(1):37-43.

17. Sun Q, Zhang Q, Gu J, et al. Prevalence, risk factors, management, and treatment outcomes of first-line antituberculous drug-induced liver injury: a prospective cohort study. Pharmacoepidemiol Drug Saf. 2016;25(8):908-917.

18. Paterson JM, Mamdani MM, Manno M, Juurlink DN, Canadian Drug Safety and Effectiveness Research Network. Fluoroquinolone therapy and idiosyncratic acute liver injury: a population-based study. CMAJ 2012;184(14):1565-1570.

19. van Bambeke F, Tulkens PM. Safety profile of the respiratory fluoroquinolone moxifloxacin: comparison with other fluoroquinolones and other antibacterial classes. Drug Saf. 2009;32(5):359-378.

20. Moreno L, Sánchez-Delgado J, Vergara M, Casas M, Miquel M, Dalmau B. Recurrent drug-induced liver injury (DILI) with ciprofloxacin and amoxicillin/clavulanic. Rev Esp Enferm Dig. 2015;107(12): 767-768.

21. Unger C, Al-Jashaami LS. Ciprofloxacin exposure leading to fatal hepatotoxicity: an unusual correlation. Am J Case Rep. 2016;17:676-681.

22. Schloss M, Becak D, Tosto ST, Velayati A. A case of LevofloxacinInduced hepatotoxicity. Am J Case Rep. 2018;19:272-276.
23. Steuerwald NM, Foureau DM, Norton HJ, et al. Profiles of serum cytokines in acute drug-induced liver injury and their prognostic significance. PLoS One. 2013;8(12):e81974.

24. Laverty HG, Antoine DJ, Benson C, Chaponda M, Williams D, Kevin Park B. The potential of cytokines as safety biomarkers for drug-induced liver injury. Eur J Clin Pharmacol. 2010;66(10):961-976.

25. Chalasani N, Björnsson E. Risk factors for idiosyncratic drug-induced liver injury. Gastroenterology. 2010;138(7):2246-2259.

26. Chalasani NP, Hayashi PH, Bonkovsky HL, Navarro VJ, Lee WM, Fontana RJ. ACG clinical guideline: the diagnosis and management of idiosyncratic drug-induced liver injury. Am J Gastroenterol. 2014; 109(7):950-966.

27. Ahmad J, Odin JA. Epidemiology and genetic risk factors of drug hepatotoxicity. Clin Liver Dis. 2017;21(1):55-72.

28. Andrade RJ, Tulkens PM. Hepatic safety of antibiotics used in primary care. J Antimicrob Chemother. 2011;66(7):1431-1446.

29. Björnsson ES, Bergmann OM, Björnsson HK, Kvaran RB, Olafsson S. Incidence, presentation, and outcomes in patients with drug-induced liver injury in the general population of Iceland. Gastroenterology. 2013;144(7):1419-1425.

30. Singla R, Sharma SK, Mohan A, et al. Evaluation of risk factors for antituberculosis treatment induced hepatotoxicity. Indian J Med Res. 2010;132:81-86

31. Babalık A, Arda H, Bakırcı N, et al. Management of and risk factors related to hepatotoxicity during tuberculosis treatment. Tuberk Toraks. 2012;60(2):136-144.

32. Chen M, Suzuki A, Borlak J, Andrade RJ, Lucena MI. Drug-induced liver injury: interactions between drug properties and host factors. J Hepatol. 2015;63(2):503-514.

33. Ortega-Alonso A, Stephens C, Lucena M, Andrade R. Case characterization, clinical features and risk factors in drug-induced liver injury. Int J Mol Sci. 2016;17(5):714.

34. Alqahtani SA, Kleiner DE, Ghabril M, et al. Identification and characterization of Cefazolin-Induced liver injury. Clin Gastroenterol Hepatol. 2015;13(7):1328-1336.e2.

35. Haque T, Sasatomi E, Hayashi PH. Drug-induced liver injury: pattern recognition and future directions. Gut Liver. 2016;10(1):27-36.

36. Urban TJ, Shen Y, Stolz A, et al. Limited contribution of common genetic variants to risk for liver injury due to a variety of drugs. Pharmacogenet Genomics. 2012;22(11):784-795.

37. Nicoletti P, Aithal GP, Bjornsson ES, et al. Association of Liver Injury From Specific Drugs, or Groups of Drugs, With Polymorphisms in HLA and Other Genes in a Genome-Wide Association Study. Gastroenterology. 2017;152(5):1078-1089.
Therapeutics and Clinical Risk Management

\section{Publish your work in this journal}

Therapeutics and Clinical Risk Management is an international, peerreviewed journal of clinical therapeutics and risk management, focusing on concise rapid reporting of clinical studies in all therapeutic areas, outcomes, safety, and programs for the effective, safe, and sustained use of medicines. This journal is indexed on PubMed Central, CAS,

\section{Dovepress}

EMBase, Scopus and the Elsevier Bibliographic databases. The manuscript management system is completely online and includes a very quick and fair peer-review system, which is all easy to use. Visit http://www.dovepress.com/testimonials.php to read real quotes from published authors. 